\title{
Solubility of Tris(hydroxymethyl)aminomethane in Methanol + 1-Propanol Mixtures at Various Temperatures
}

\author{
Vahid Jouyban-Gharamaleki, ${ }^{\dagger}$ Karim Jouyban-Gharamaleki, ${ }^{\ddagger}$ Jafar Soleymani, ${ }^{\S}$ Ernst Kenndler,"
} William E. Acree, Jr., ${ }^{\perp}$ and Abolghasem Jouyban $* \#, \nabla$

${ }^{\dagger}$ Department of Mechatronic Engineering, International Campus, University of Tabriz, Tabriz, Iran

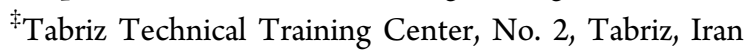

${ }^{\S}$ Liver and Gastrointestinal Diseases Research Center, Tabriz University of Medical Sciences, Tabriz 51664, Iran

"Institute for Analytical Chemistry, Faculty of Chemistry, University of Vienna, Währingerstrasse 38, A 1090 Vienna, Austria

${ }^{\perp}$ Department of Chemistry, University of North Texas, Denton, Texas 76203-5070, United States

\#Drug Applied Research Center and Faculty of Pharmacy, Tabriz University of Medical Sciences, Tabriz 51664, Iran

${ }^{\nabla}$ Pharmaceutical Engineering Laboratory, School of Chemical Engineering, College of Engineering, University of Tehran, P.O. Box 11155/4563, Tehran, Iran

ABSTRACT: Solubility of tris(hydroxymethyl)aminomethane (TRIS) in various mass fractions of binary solvent mixtures of methanol and 1-propanol [at (293.2, 298.2, 303.2, 308.2, and 313.2) $\mathrm{K}$ ] was measured and mathematically represented using the Jouyban-Acree model. The correlated mole fraction solubilities are in good agreement with the corresponding experimental values with an overall mean percentage deviation of $1.7 \%$. Densities of saturated solutions of TRIS in pure methanol and 1-propanol at various temperatures were measured and employed to predict the density of saturated solutions of TRIS in their binary solvent mixtures.

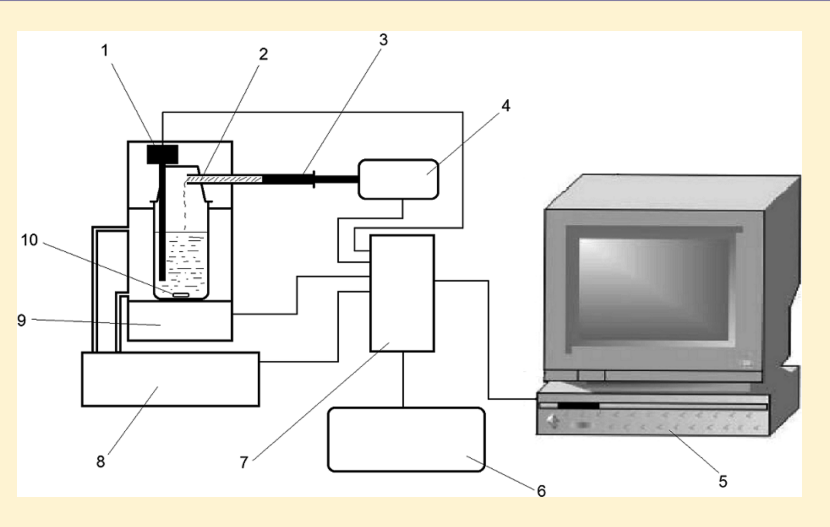

\section{INTRODUCTION}

Buffer solutions composed of binary solvents are used as mobile phases and/or background electrolytes in analytical separation methods such as liquid chromatography or capillary electrophoresis, where the low solubility of the buffering agents at higher concentrations of the low polar solvent may cause practical problems. Using nonaqueous based mobile phases and/or running buffers, suitable analytical methods enable analyzing highly hydrophobic analytes or compounds that exhibit restricted stability in aqueous media. ${ }^{1}$ Addition of the second organic solvent to aqueous solutions alters the solubility of the analyte, the resolution of analytes, or determine other relevant analytical parameters like $\mathrm{p} K_{\mathrm{a}}$ values, partition coefficients or electrophoretic mobilities. ${ }^{2,3}$ In addition to these applications, the solubility data of TRIS is also required in solubilization/crystallization studies. The common method for obtaining a solute's solubility is the trial and error approach which is a time-consuming and costly method. Any predictive tool to estimate the best solvent composition for solubilization/ crystallization of Tris(hydroxymethyl)aminomethane (TRIS) is obviously in demand.

TRIS (chemical abstract service number 77-86-1) is a common buffering agent in chemical and biochemical areas. It is used in nonaqueous solvents for analytical and separation purposes. Available solubility data of TRIS in various solvent mixtures is reviewed in a recent publication ${ }^{4}$ along with reporting TRIS solubility in water + methanol $(\mathrm{MeOH})^{2}$ and water + 1-propanol $(1-\mathrm{PrOH})^{5}$ mixtures at 293.2 to $313.2 \mathrm{~K}$. A quick survey on the published pharmaceutical analysis methods showed that $\mathrm{MeOH}+1-\mathrm{PrOH}$ has been used in some developed analytical methods. ${ }^{6}$

To continue our systematic investigations on the solubility of solutes in solvent mixtures, the solubility of TRIS in $\mathrm{MeOH}+$ 1-PrOH mixtures at different temperatures [(293.2, 298.2, $303.2,308.2$, and 313.2$) \mathrm{K}$ ] was measured using a validated labmade setup., ${ }^{4,7}$ The trained version of a mathematical model is presented to provide a predictive tool for calculation of the solubility of TRIS at any composition of $\mathrm{MeOH}+1-\mathrm{PrOH}$ solvent mixture and at any temperature in the range mentioned.

\section{EXPERIMENTAL SECTION}

Materials. TRIS (with the stated purity of 0.999 in mass fraction) and $\mathrm{MeOH}$ (mass fraction purity of 0.999) were purchased from Merck (Germany). 1-PrOH (mass fraction

Received: October 19, 2014

Accepted: November 15, 2014

Published: November 26, 2014 
Table 1. List of the Used Materials

\begin{tabular}{|c|c|c|c|}
\hline material & purity/in mass fraction & company & country \\
\hline TRIS: tris(hydroxymethyl)aminomethane (CASRN 77-86-1) & 0.999 & Merck & Germany \\
\hline $\mathrm{MeOH}$ & 0.999 & Merck & Spain \\
\hline 1-PrOH & 0.999 & Scharlau & Germany \\
\hline
\end{tabular}

Table 2. Experimental Mole Fraction Solubility ${ }^{a}$ of Tris(hydroxymethyl)aminomethane (TRIS) in Binary Mixtures of MeOH $(1)+1-\mathrm{PrOH}(2)$ at Various Temperatures ${ }^{b}$

$\begin{array}{ccccccccccccc}x_{1}^{0 c} & 293.2 \mathrm{~K} & \mathrm{SD} & 298.2 \mathrm{~K} & \mathrm{SD} & 303.2 \mathrm{~K} & \text { SD } & 308.2 \mathrm{~K} & \text { SD } & 313.2 \mathrm{~K} & \text { SD } & & \\ 1.00 & 7.60 \times 10^{-3} & 4.00 \times 10^{-4} & 9.20 \times 10^{-3} & 3.00 \times 10^{-4} & 1.06 \times 10^{-2} & 6.00 \times 10^{-4} & 1.24 \times 10^{-2} & 6.00 \times 10^{-4} & 1.58 \times 10^{-2} & 7.00 \times 10^{-4} \\ 0.94 & 7.10 \times 10^{-3} & 2.00 \times 10^{-4} & 8.60 \times 10^{-3} & 3.00 \times 10^{-4} & 1.03 \times 10^{-2} & 3.00 \times 10^{-4} & 1.21 \times 10^{-2} & 2.00 \times 10^{-5} & 1.52 \times 10^{-2} & 1.00 \times 10^{-4} \\ 0.88 & 6.40 \times 10^{-3} & 1.00 \times 10^{-4} & 7.80 \times 10^{-3} & 3.00 \times 10^{-4} & 9.30 \times 10^{-3} & 3.00 \times 10^{-4} & 1.12 \times 10^{-2} & 3.00 \times 10^{-4} & 1.38 \times 10^{-2} & 5.00 \times 10^{-4} \\ 0.81 & 5.80 \times 10^{-3} & 1.00 \times 10^{-4} & 6.90 \times 10^{-3} & 1.00 \times 10^{-4} & 8.30 \times 10^{-3} & 3.00 \times 10^{-4} & 1.00 \times 10^{-2} & 3.00 \times 10^{-4} & 1.25 \times 10^{-2} & 5.00 \times 10^{-4} \\ 0.74 & 5.30 \times 10^{-3} & 1.00 \times 10^{-4} & 6.40 \times 10^{-3} & 2.00 \times 10^{-4} & 7.70 \times 10^{-3} & 2.00 \times 10^{-4} & 9.20 \times 10^{-3} & 1.00 \times 10^{-4} & 1.12 \times 10^{-2} & 1.00 \times 10^{-4} \\ 0.65 & 5.00 \times 10^{-3} & 2.00 \times 10^{-4} & 6.00 \times 10^{-3} & 2.00 \times 10^{-4} & 7.20 \times 10^{-3} & 3.00 \times 10^{-4} & 8.70 \times 10^{-3} & 4.00 \times 10^{-4} & 1.04 \times 10^{-2} & 5.00 \times 10^{-4} \\ 0.56 & 4.40 \times 10^{-3} & 3.00 \times 10^{-5} & 5.40 \times 10^{-3} & 1.00 \times 10^{-4} & 6.70 \times 10^{-3} & 4.00 \times 10^{-5} & 8.00 \times 10^{-3} & 1.00 \times 10^{-4} & 9.80 \times 10^{-3} & 1.00 \times 10^{-4} \\ 0.45 & 4.30 \times 10^{-3} & 1.00 \times 10^{-4} & 5.30 \times 10^{-3} & 1.00 \times 10^{-4} & 6.60 \times 10^{-3} & 2.00 \times 10^{-4} & 7.80 \times 10^{-3} & 1.00 \times 10^{-4} & 9.70 \times 10^{-3} & 4.00 \times 10^{-4} \\ 0.32 & 4.10 \times 10^{-3} & 1.00 \times 10^{-4} & 5.20 \times 10^{-3} & 1.00 \times 10^{-4} & 6.50 \times 10^{-3} & 1.00 \times 10^{-4} & 7.70 \times 10^{-3} & 2.00 \times 10^{-4} & 9.60 \times 10^{-3} & 4.00 \times 10^{-4} \\ 0.17 & 3.90 \times 10^{-3} & 2.00 \times 10^{-4} & 5.10 \times 10^{-3} & 4.00 \times 10^{-5} & 6.40 \times 10^{-3} & 1.00 \times 10^{-4} & 7.40 \times 10^{-3} & 2.00 \times 10^{-5} & 9.20 \times 10^{-3} & 6.00 \times 10^{-4} \\ 0.00 & 3.80 \times 10^{-3} & 2.00 \times 10^{-4} & 5.00 \times 10^{-3} & 2.00 \times 10^{-4} & 6.30 \times 10^{-3} & 1.00 \times 10^{-4} & 7.20 \times 10^{-3} & 4.00 \times 10^{-5} & 8.80 \times 10^{-3} & 1.00 \times 10^{-4}\end{array}$

${ }^{a}$ Data are the mean of three measurements. SD: standard deviation. ${ }^{b}$ The relative standard uncertainty for the solubilities is $2.6 \%$ or ur $(x)=0.026$, the standard uncertainty for temperature is $0.1 \mathrm{~K}$ and the measurements were made at atmospheric pressure. ${ }^{c} x_{1}^{0}$ is the mole fractions of MeOH in the absence of TRIS.

purity of 0.999) was purchased from Scharlau (Spain). All reagents were used without further purifications. Table 1 lists the details of materials used in this work.

Instrumentation. The solute solubility is determined employing various techniques which are reviewed in a recent work. ${ }^{8}$ The common so-called synthetic method ${ }^{9}$ is based on the disappearance of the solid solute from the solution as monitored by a laser beam. A lab-made setup was applied for the measurement of the solubility of TRIS in $\mathrm{MeOH}+1-\mathrm{PrOH}$ mixtures. It is based on this synthetic method and is described in earlier works. ${ }^{4,7,10}$ Briefly, a syringe filled with TRIS powder was weighed using an electronic balance (Sartorius, Germany) with uncertainty of $0.01 \mathrm{~g}$ before and after saturation of the solution (the mass difference determines the mass of solute dissolved to saturate the solution). In more detail, after reaching the experimental temperature, the setup added a small amount of TRIS to a given mass of the solvent and/or solvent mixture, and the content was stirred continuously at a constant temperature with the uncertainty of $\pm 0.1 \mathrm{~K}$. As the particles of TRIS were dissolved, the signals indicating the number of particles decreased gradually and reached to the minimum value when TRIS is completely dissolved. Then an additional amount of TRIS was dispensed to the vessel and the procedure was repeated until the laser beam could not return to the minimum value; an indication that the last added portion of powder could not be dissolved. This cycle was checked several times by the software, and then the system was stopped and the total amount of the added drug was recorded and used to calculate the solubility value. The signals for pure solvents are considered as the minimum intensity of the signals detected by the photo converter. Further details of the setup have been provided in earlier works. ${ }^{4,10}$ It should be noted that the total masses of the solvent mixtures were $120 \mathrm{~g}$, and those of the solute plus the syringe varied between $(0.10$ and 4.00$) \mathrm{g}$. All measurements were conducted at atmospheric pressure.

Calculations. The average of measurements carried out at least in triplicate was used to calculate the mole fraction solubility of TRIS. The masses $\left(w_{1}\right.$ and $\left.w_{2}\right)$ of solvents 1
$(\mathrm{MeOH})$ and $2(1-\mathrm{PrOH})$ were divided by their molar masses $\left(\mathrm{MW}_{1}\right.$ and $\left.M \mathrm{MW}_{2}\right)$, and then the mole fractions of solvents 1 and 2 in the absence of the solute $\left(x_{1}^{0}\right.$ and/or $\left.x_{2}^{0}\right)$ were computed as

$$
x_{i}^{0}=\frac{\frac{w_{i}}{\mathrm{MW}_{i}}}{\left(\frac{w_{1}}{\mathrm{MW}_{1}}+\frac{w_{2}}{\mathrm{MW}_{2}}\right)}
$$

The saturated mole fraction solubility of TRIS $\left(x_{m, T}\right)$ at different compositions of the binary $\mathrm{MeOH}+1-\mathrm{PrOH}$ mixtures at various temperatures was calculated using

$$
x_{m, T}=\frac{\frac{w_{\mathrm{TRIS}}}{\mathrm{MW}_{\mathrm{TRIS}}}}{\left(\frac{w_{\mathrm{TRIS}}}{\mathrm{MW}_{\mathrm{TRIS}}}+\frac{w_{1}}{\mathrm{MW}_{1}}+\frac{w_{2}}{\mathrm{MW}_{2}}\right)}
$$

where $w_{\text {TRIS }}$ and $\mathrm{MW}_{\text {TRIS }}$ are the mass of TRIS added to saturate the solution and the molar mass of TRIS, respectively. Evaluation of the accuracy of the calculated data was performed by computing the mean percentage deviations (MPD) between calculated and experimental solubilities according to

$$
\mathrm{MPD}=\frac{100}{N} \sum\left(\frac{x_{m, T}^{\text {calculated }}-x_{m, T}^{\text {experimental }}}{x_{m, T}^{\text {experimental }}}\right)
$$

where $N$ is the number of data points in each set.

\section{RESULTS AND DISCUSSION}

Table 2 lists the experimental mole fraction solubility of TRIS at various mole fractions of $\mathrm{MeOH}(1)+1-\mathrm{PrOH}(2)$ mixtures at the temperatures investigated. The solubility of TRIS in the monosolvents and at each solvent composition increased with increasing temperature. This is an expected solubility pattern for such type of solid solutes in the solutions. The mole fraction solubility of TRIS in a given solvent system at various temperatures $\left(x_{T}\right)$ could be represented using the van't Hoff equation ${ }^{11}$ 


$$
\ln x_{T}=A+\frac{B}{T}
$$

where $A$ and $B$ are the model constants calculated using a leastsquare method. Table 3 lists these model constants and the

Table 3. Model Constants for van't Hoff Equation for Various Mole Fractions of $\mathrm{MeOH}\left(x_{1}^{0}\right)$ and the obtained Mean Percentage Deviation (MPD) for Back-Calculated Solubilities Using eq 4

$\begin{array}{cccc}x_{1}^{0} & \mathrm{~A} & \mathrm{~B} & \mathrm{MPD} \\ 1.00^{a} & 6.07 & -3212 & 2.4 \\ 0.94 & 6.74 & -3426 & 1.4 \\ 0.88 & 6.81 & -3478 & 1.2 \\ 0.81 & 6.87 & -3531 & 1.8 \\ 0.74 & 6.23 & -3361 & 0.7 \\ 0.65 & 6.32 & -3408 & 0.4 \\ 0.56 & 6.04 & -3653 & 0.6 \\ 0.45 & 7.10 & -3679 & 0.7 \\ 0.32 & 7.67 & -3864 & 1.3 \\ 0.17 & 7.45 & -3812 & 2.1 \\ 0.00^{b} & 7.22 & -3740 & 3.2 \\ & & \text { overall } & 1.4\end{array}$

${ }^{a}$ Data for this fraction taken from a previous work. ${ }^{4}$ Data for this fraction taken from a previous work. ${ }^{5}$

MPD values for the back-calculated data using eq 4. Good linear relationship between $\ln x_{T}$ and $1 / T$ is found for the temperature range in this study.

Using the combined nearly ideal binary solvent/RedlichKister equation, ${ }^{12}$ the solubility of TRIS in $\mathrm{MeOH}(1)+1$ $\mathrm{PrOH}$ (2) mixtures at a given temperature could be represented as

$$
\ln x_{m}=x_{1}^{0} \ln x_{1}+x_{2}^{0} \ln x_{2}+x_{1}^{0} x_{2}^{0}\left[\sum_{i=0}^{2} S_{i}\left(x_{1}^{0}-x_{2}^{0}\right)^{i}\right]
$$

where $x_{m}, x_{1}$, and $x_{2}$ are the mole fraction solubility of TRIS at a given temperature in the solvent mixtures and the monosolvents 1 and $2 ; S_{i}$ are the constants of the model calculated using a no intercept least-square analysis. ${ }^{13}$ Table 4 lists the numerical values of the model constants of eq 5 and the obtained MPD values for the back-calculated solubility data.

Table 4. Model Constants of eq 5 and the MPD Values for Back-Calculated Solubilities in $\mathrm{MeOH}+1-\mathrm{PrOH}$ Mixtures at Various Temperatures (K)

\begin{tabular}{ccccc}
$T / \mathrm{K}$ & $S_{0}$ & $S_{1}$ & $S_{2}$ & $\mathrm{MPD}$ \\
293.2 & -247.2 & -69.5 & 110.6 & 1.0 \\
298.2 & -292.8 & -102.3 & 122.4 & 1.0 \\
303.2 & -264.1 & -95.5 & 165.4 & 1.3 \\
308.2 & -227.1 & -61.5 & 205.2 & 1.5 \\
313.2 & -256.2 & -143.5 & 256.2 & 1.5 \\
& & & overall & 1.3 \\
\hline
\end{tabular}

The model has been extended to represent both solvent composition and temperature effects on a solute's solubility as ${ }^{14}$

$$
\ln x_{m, T}=x_{1}^{0} \ln x_{1, T}+x_{2}^{0} \ln x_{2, T}+\frac{x_{1}^{0} x_{2}^{0}}{T}\left[\sum_{i=0}^{2} J_{i}\left(x_{1}^{0}-x_{2}^{0}\right)^{i}\right]
$$

where $x_{m, T}$ is the mole fraction solubility in the solvent mixtures at temperature $T$ (in degree $K$ ), $x_{1, T}$ and $x_{2, T}$ are the mole fraction solubility of TRIS in the monosolvents 1 and 2, respectively, and $J_{i}$ stands for the constants of the model computed by a regression analysis. ${ }^{13}$ The solubility data at all temperatures were fitted to eq 6 , and the obtained model is

$$
\begin{aligned}
\ln x_{m, T}= & x_{1}^{0} \ln x_{1, T}+x_{2}^{0} \ln x_{2, T}+\frac{x_{1}^{0} x_{2}^{0}}{T} \\
& {\left[-257.792-93.734\left(x_{1}^{0}-x_{2}^{0}\right)\right.} \\
& \left.+169.501\left(x_{1}^{0}-x_{2}^{0}\right)^{2}\right]
\end{aligned}
$$

which correlates the solubility data with linear correlation coefficient of 0.986 with $p<0.0005$. The obtained MPD value for the back-calculated solubility data was $1.7 \pm 1.5 \%(N=55)$.

The Jouyban-Acree model was combined with the van't Hoff model as ${ }^{4,15,16}$

$$
\begin{aligned}
\ln x_{m, T}= & x_{1}^{0}\left(A_{1}+\frac{B_{1}}{T}\right)+x_{2}^{0}\left(A_{2}+\frac{B_{2}}{T}\right) \\
& +\frac{x_{1}^{0} x_{2}^{0}}{T}\left[\sum_{i=0}^{2} J_{i}\left(x_{1}^{0}-x_{2}^{0}\right)^{i}\right]
\end{aligned}
$$

to provide a more versatile predictive equation. The model is obtained from combining the trained van't Hoff equations using $x_{1}^{0}=0.00$ and $x_{1}^{0}=1.00$ and the $J$ terms from eq 7 as

$$
\begin{aligned}
\ln x_{m, T}= & x_{1}^{0}\left(6.069-\frac{3211.508}{T}\right) \\
& +x_{2}^{0}\left(7.224-\frac{3740.048}{T}\right)+\frac{x_{1}^{0} x_{2}^{0}}{T} \\
& {\left[-257.792-93.734\left(x_{1}^{0}-x_{2}^{0}\right)\right.} \\
& \left.+169.501\left(x_{1}^{0}-x_{2}^{0}\right)^{2}\right]
\end{aligned}
$$

which enables the back-calculation of the solubility data with the MPD of $2.0 \pm 1.6 \%(N=55)$, nearly the same as obtained applying eq 7 .

The densities of the saturated solutions of the solute are required to convert mole fraction solubility to molar solubility or vice versa. The following equation could be employed to predict the density of saturated solutions of TRIS in $\mathrm{MeOH}$ (1) $+1-\mathrm{PrOH}$ (2) using

$$
\begin{aligned}
\ln \rho_{m, T}^{\text {sat }}= & x_{1}^{0} \ln \rho_{1, T}^{\text {sat }}+x_{2}^{0} \ln \rho_{2, T}^{\text {sat }}+\frac{x_{1}^{0} \cdot x_{2}^{0}}{T} \\
& {\left[1.927+0.204\left(x_{1}^{0}-x_{2}^{0}\right)-0.504\left(x_{1}^{0}-x_{2}^{0}\right)^{2}\right] }
\end{aligned}
$$

in which $\rho_{m, T}^{\text {sat }}$ is the density of the saturated solution of TRIS in the mixed solvent system and $\rho_{1, T}^{\text {sat }}$ and $\rho_{2, T}^{\text {sat }}$ are the density of saturated solutions of TRIS in the monosolvents 1 and 2 at different temperatures. The model constants of eq 10 were derived from fitting the experimental density of the solute free mixtures of $\mathrm{MeOH}+1-\mathrm{PrOH}$ at various temperatures collected from the literature. ${ }^{17}$ Full details of this procedure has been described in an earlier work. ${ }^{5}$ The measured values for $\rho_{1, T}^{\text {sat }}$ and $\rho_{2, T}^{\text {sat }}$ of the saturated solutions of TRIS in $\mathrm{MeOH}$ and $1-\mathrm{PrOH}$ at various temperatures along with their solute-free values ${ }^{17}$ are listed in Table 5. The patterns for the densities of $\mathrm{MeOH}$ and $1-\mathrm{PrOH}$ in the absence of TRIS decreased due to volume expansion of the solvents at higher temperatures. Using eq 10, 
Table 5. Density of TRIS Saturated Solutions of TRIS in $\mathrm{MeOH}$ and 1-PrOH at Various Temperatures, and Corresponding Solute Free Density Values Taken from a Paper $^{17}$ (Reported in Parentheses)

\begin{tabular}{cccc}
$T$ & $\rho / \mathrm{g} \cdot \mathrm{cm}^{-3}$ & & $\rho / \mathrm{g} \cdot \mathrm{cm}^{-3}$ \\
\cline { 1 - 2 } 293.2 & $\mathrm{MeOH}$ & $1-\mathrm{PrOH}$ \\
298.2 & $0.8380(0.7913)$ & & $0.8247(0.8043)$ \\
303.2 & $0.8420(0.7867)$ & & $0.8227(0.8002)$ \\
308.2 & $0.8440(0.7820)$ & & $0.8218(0.7964)$ \\
313.2 & $0.8460(0.7774)$ & & $0.8187(0.7923)$ \\
& $0.8480(0.7728)$ & & $0.8180(0.7885)$ \\
\hline
\end{tabular}

densities of saturated solutions of TRIS in $\mathrm{MeOH}+1-\mathrm{PrOH}$ mixtures at various temperatures [at (293.2 to 313.2$) \mathrm{K}$ ] can be predicted with acceptable accuracies as shown in earlier works. $^{18-23}$

\section{CONCLUSIONS}

The experimental solubilities of TRIS in binary mixtures of $\mathrm{MeOH}+1-\mathrm{PrOH}$ at $(293.2,298.2,303.2,308.2$, and 313.2) $\mathrm{K}$ were measured and used to provide a trained version of the Jouyban-Acree model combined with the van't Hoff equation to predict the solubility of TRIS at any composition of the $\mathrm{MeOH}$ $+1-\mathrm{PrOH}$ mixtures at any temperature of interest [between (293.2 and 313.2) K]. This work extends the available solubility database of solutes in mixed solvents. ${ }^{24}$ The results can be employed for practical purposes, e.g., for crystallization procedures in the chemical industry or solubilization requirements for methods like liquid chromatography or capillary electrophoresis. A numerical method was reported for predicting the density of saturated solutions of TRIS in $\mathrm{MeOH}+1-\mathrm{PrOH}$ mixtures.

\section{AUTHOR INFORMATION}

\section{Corresponding Author}

* E-mail: ajouyban@hotmail.com and jouyban@ut.ac.ir. Fax: +984133363231.

\section{Notes}

The authors declare no competing financial interest.

\section{REFERENCES}

(1) Porras, S.; Kenndler, E. Capillary Zone Electrophoresis in NonAqueous Solutions: $\mathrm{pH}$ of the Background Electrolyte. J. Chromatogr. A 2004, 1037, 455-465.

(2) Anubala, S.; Sekar, R; Nagaiah, K. Development and Validation of An Analytical Method for the Separation and Determination of Major Bioactive Curcuminoids in Curcuma Longa Rhizomes and Herbal Products Using Non-Aqueous Capillary Electrophoresis. Talanta 2014, 123, 10-17.

(3) Jouyban, A.; Kenndler, E. Capillary Electrophoresis with Organic Solvents in Pharmaceutical Analysis: A Systematic Guide Through the Background. Cur. Anal. Chem. 2014, 10, 248-266.

(4) Jouyban-Gharamaleki, V.; Jouyban-Gharamaleki, K.; Soleymani, J.; Acree, W. E., Jr.; Jouyban, A. Solubility Determination of Tris(hydroxymethyl)aminomethane in Water + Methanol Mixtures at Various Temperatures Using a Laser Monitoring Technique. J. Chem. Eng. Data 2014, 59, 2305-2309.

(5) Jouyban-Gharamaleki, V.; Jouyban-Gharamaleki, K.; Soleymani, J.; Acree, W. E., Jr.; Jouyban, A. Solubility Determination of Tris(hydroxymethyl)aminomethane in Water + 1-Propanol Mixtures at Various Temperatures. J. Chem. Eng. Data 2014, in press, DOI:10.1021/je500620m.
(6) Lunn, G. HPLC Methods for Pharmaceutical Analysis. John Wiley and Sons, Inc.: New York, 2000.

(7) Jouyban-Gharamaleki, V.; Jouyban-Gharamaleki, K.; Shayanfar, A.; Khoubnasabjafari, M.; Jouyban, A. An Automated System for Determination of Drug Solubility Based on Laser Monitoring Technique. J. Lab. Auto. 2014, in press, doi:10.1177/ 2211068214554801.

(8) Jouyban, A.; Fakhree, M. A. A. In Toxicity and Drug Testing; Acree, W. E., Jr., Ed.; InTech Publisher: New York, 2012.

(9) Hankinson, R. W.; Thompson, D. Equilibria and Solubility Data for Methanol - Water-1-Nitropropane Ternary Liquid System. J. Chem. Eng. Data 1965, 10, 18-19.

(10) Jouyban-Gharamaleki, K.; Jouyban-Gharamaleki, V.; Jouyban, A. An Automated Solubility Setup. Iranian Patent Office, 78899, 2013.

(11) Grant, D. J. W.; Mehdizadeh, M.; Chow, A. H. L.; Fairbrother, J. E. Non-Linear van't Hoff Solubility-Temperature Plots and Their Pharmaceutical Interpretation. Int. J. Pharm. 1984, 18, 25-38.

(12) Acree, W. E., Jr. Mathematical Representation of Thermodynamic Properties. Part II. Derivation of the Combined Nearly Ideal Binary Solvent (NIBS)/Redlich-Kister Mathematical Representation from a Two-Body and Three-Body Interactional Mixing Model. Thermochim. Acta 1992, 198, 71-79.

(13) Jouyban-Gharamaleki, A.; Hanaee, J. A Novel Method for Improvement of Predictability of the CNIBS/R-K Equation. Int. J. Pharm. 1997, 154, 245-247.

(14) Jouyban-Gharamaleki, A.; Acree, W. E., Jr. Comparison of Models for Describing Multiple Peaks in Solubility Profiles. Int. J. Pharm. 1998, 167, 177-182.

(15) Jouyban, A.; Fakhree, M. A. A.; Acree, W. E., Jr. Comment on "Measurement and Correlation of Solubilities of (Z)-2-(2-Aminothiazol-4-yl)-2-methoxyiminoacetic Acid in Different Pure Solvents and Binary Mixtures of Water + (Ethanol, Methanol, or Glycol). J. Chem. Eng. Data 2012, 57, 1344-1346.

(16) Sardari, F.; Jouyban, A. Solubility of Nifedipine in Ethanol + Water and Propylene Glycol + Water Mixtures at 293.2 to $313.2 \mathrm{~K}$. Ind. Eng. Chem. Res. 2013, 52, 14353-14358.

(17) Borun, A.; Zurada, M.; Bald, A. Densities and Excess Molar Volumes for Mixtures of Methanol with other Alcohols at Temperatures (288.15-313.15 K). J. Therm. Anal. Calorim. 2010, 100, 707715 .

(18) Soltanpour, Sh.; Jouyban, A. Solubility of Acetaminophen and Ibuprofen in Polyethylene Glycol 600, N-Methyl Pyrrolidone and Water Mixtures. J. Solution Chem. 2011, 40, 2032-2045.

(19) Jouyban, A.; Soltanpour, Sh.; Acree, W. E., Jr. Solubility of Acetaminophen and Ibuprofen in the Mixtures of Polyethylene Glycol 200 or 400 with Ethanol and Water and the Density of Solute-Free Mixed Solvents at 298.2 K. J. Chem. Eng. Data 2010, 55, 5252-5257.

(20) Soltanpour, Sh.; Jouyban, A. Solubility of Acetaminophen and Ibuprofen in Polyethylene Glycol 600, Propylene Glycol and Water Mixtures at $25{ }^{\circ} \mathrm{C}$. J. Mol. Liq. 2010, 155, 80-84.

(21) Jouyban, A. Soltanpour, Sh. Solubility of Acetaminophen and Ibuprofen in Binary and Ternary Mixtures of Polyethylene Glycol 600, Ethanol and Water. Chem. Pharm. Bull. 2010, 58, 219-224.

(22) Soltanpour, Sh.; Jouyban, A. Solubility of Lamotrigine in Binary and Ternary Mixtures of N-Methyl Pyrrolidone and Water with Polyethylene Glycols 200, 400, and 600 at 298.2 K. J. Mol. Liq. 2013, $180,1-6$.

(23) Soleymani, J.; Djozan, Dj.; Martínez, F.; Jouyban, A. Solubility of Ranitidine Hydrochloride in Solvent Mixtures of PEG 200, PEG 400, Ethanol and Propylene Glycol at $25^{\circ} \mathrm{C}$. J. Mol. Liq. 2013, 182, 91-94.

(24) Jouyban, A. Handbook of Solubility Data for Pharmaceuticals; CRC Press: Boca Raton, FL, 2010. 